\title{
Crystal Structure of Actinide Metals at High Compression
}

\author{
Lars Fast \\ Per Soderlind
}

This was prepared for submittal to the American Physical Society Topical Conference Seattle, Washington August 13-18, 1995

August 1995

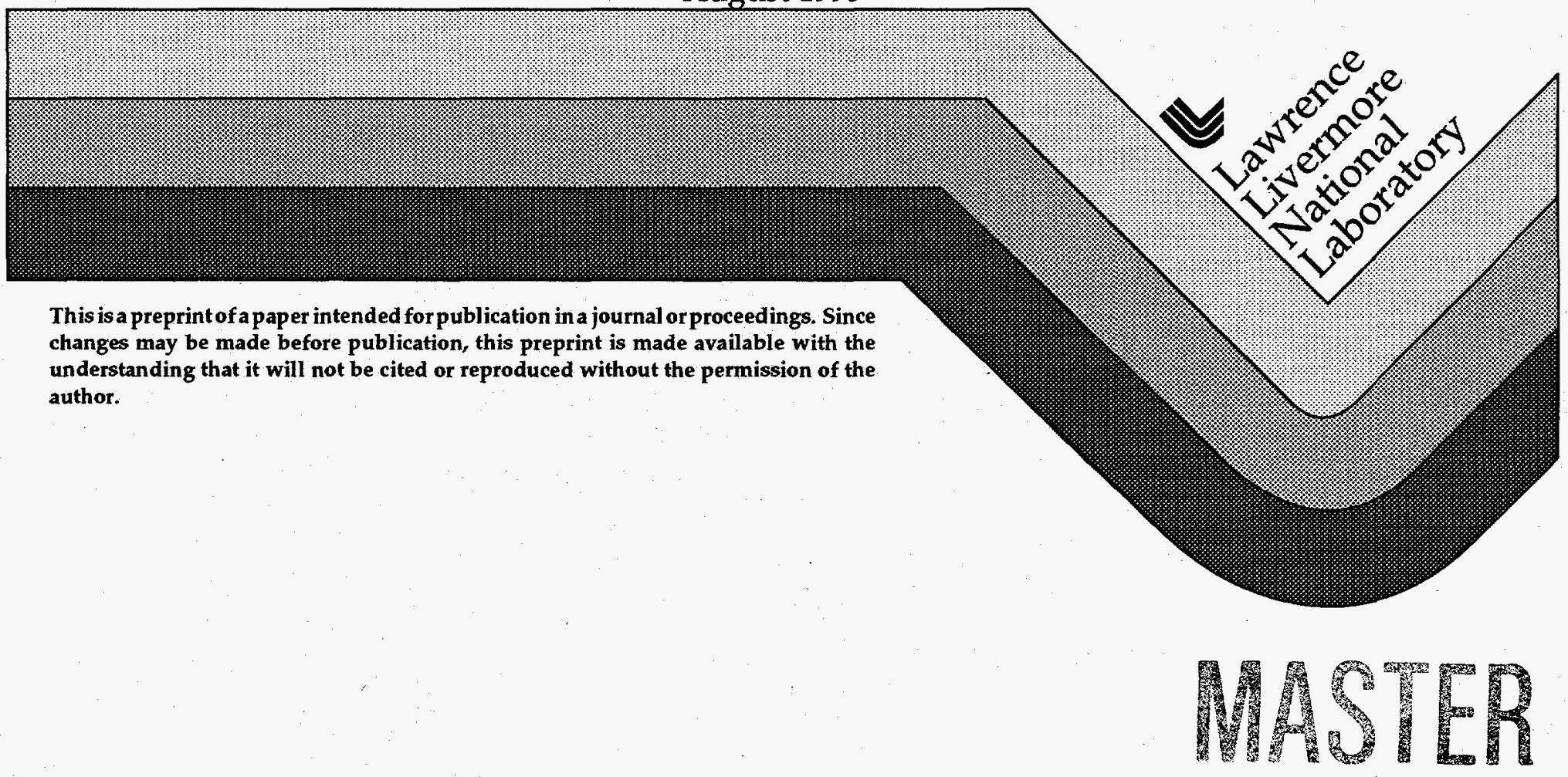




\section{DISCLAIMER}

This document was prepared as an account of work sponsored by an agency of the United States Government. Neither the United States Government nor the University of California nor any of their employees, makes any warranty, express or implied, or assumes any legal liability or responsibility for the accuracy, completeness, or usefulness of any information, apparatus, product, or process disclosed, or represents that its use would not infringe privately owned rights. Reference herein to any specific commercial product, process, or service by trade name, trademark, manufacturer, or otherwise, does not necessarily constitute or imply its endorsement, recommendation, or favoring by the United States Government or the University of California. The views and opinions of authors expressed herein do not necessarily state or reflect those of the United States Government or the University of California, and shall not be used for advertising or product endorsement purposes. 


\section{DISCLAIMER}

Portions of this document may be illegible in electronic image products. Images are produced from the best available original document. 


\title{
CRYSTAL STRUCTURE OF ACTINIDE METALS AT HIGH COMPRESSION
}

\author{
Lars Fast \\ Condensed Matter Theory Group, Physics Department, Uppsala University, Box 530. Sweden \\ Per Söderlind
}

Physics Department, Lawrence Livermore National Laboratory, Livermore, CA 94550

\begin{abstract}
The crystal structures of some light actinide metals are studied theoretically as a function of applied pressure. The first principles electronic structure theory is formulated in the framework of density functional theory, with the gradient corrected local density approximation of the exchangecorrelation functional.

The light actinide metals are shown to be well described as itinerant (metallic) f-electron metals and generally, they display a crystal structure which have, in agreement

with previous theoretical suggestions, increasing degree of symmetry and closed-packing upon compression. The theoretical calculations agree well with available experimental data. At very high compression, the theory predicts closed-packed structures such as the foc or the hep structures or the nearly closed-packed bcc structure for the light actinide metals. A simple canonical band picture is presented to explain in which particular closed-packed form these metals will crystallize at ultra-high pressure.
\end{abstract}

\section{INTRODUCTION}

The light actinide metals, $90^{\mathrm{Th}}-94^{\mathrm{Pu}}$, have cohesive properties that resemble the $d$-transition elements, with the difference that it is the $5 f$ electrons rather than the $d$ electrons that determine the cohesion. The equilibrium volume, bulk modulus, and cohesive energy behave similarly for these metals, and the light actinides are understood to be part of a $f$-transition series. However, the crystal structures for the $d$-transition and $f$-transition metals are very different. The $d$-transition metals crystallize in the closed-packed structures fcc and hcp or the nearly closed-packed bcc structure, all with a high degree of symmetry. These structures are in sharp contrast to the anomalous crystal structures that the early actinides prefer, which are open/complex with very low symmetry. Clearly this behavior is dictated by the bonding properties of the $5 f$ electrons in these metals, but a good understanding of the mechanism involved has only recently been presented (1). As an accurate description of this mechanism has evolved, a better understanding of the high pressure phases of the actinides has been possible.

In the following sections we present our computational technique, the symmetry breaking mechanism that stabilize the light actinides in their crystal structures at ambient conditions, and a simple canonical band model, which can be used to understand the crystal structures for these metals at high compression.

\section{COMPUTATIONAL DETAILS}

The electronic structure method used to calculate the total energy for the actinides is based on the density functional theory (2). Usually a local approximation of the exchange-correlation energyfunctional is used, but we have also taken into account the gradient of the charge density in our calculations (3), (4). The theory is implemented with

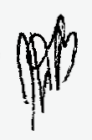


the linear muffin-tin orbital method for a full potential (FP-LMTO) (5). With this accurate approach, we have been able to calculate the total energy for the actinides in their highly distorted crystal structures. This would not have been possible with methods that rely upon spherical approximations of the electron charge density and/or potential.

Our full potential technique requires that the crystal is divided into non-overlapping muffin-tin spheres surrounded by an interstitial region. The muffin-tin spheres and the interstitial region are then treated differently; Inside the muffin-tin spheres the basis functions are expanded in muffin-tin orbitals (6) and the basis set consists of $6 s, 6 p, 7 s, 7 p, 6 d$, and $5 f$ partial waves for which a separate set of energy parameters are used for the two first, lower lying, semi-core states. Two or three tail functions with different kinetic energies $\kappa^{2}$ are attached to each basis function, i.e., a "double" or "triple" basis is used. The radial part of these functions outside the muffin-tin spheres is a solution to Helmholtz equation, i.e., Neumann or Hankel functions (depending on the sign of $\kappa^{2}$ ) whereas the angular part is a spherical harmonic function. These functions are represented in a Fourier series in the interstitial region. The special $k$-point method is used in the k-point sampling within the Brillouin zone, with a gaussian smearing of $20 \mathrm{mRy}$ for each k-point.

\section{RESULTS}

The first actinide metal, with an appreciable $5 f$ occupation is thorium. At equilibrium conditions the number of $5 f$ electrons are- relatively small, but as the hydrostatic pressure increases, the $5 f$ population increases (7) and consequently a distorted (bct) structure is found experimentally at about $75 \mathrm{GPa}$ (8). This fec-bet transition has been studied earlier and theoretically (4) the c/a value of the bct structure, as a function of applied pressure, is in a very good agreement with experiment (8). As Th is compressed further, we predict another crystallographic transition from bet to the hep structure at approximately $8 \AA^{3}$, see Fig.1. For the hcp structure the cla ratio is close to the ideal value, in the volume interval examined.

For the rest of the light actinides, Pa-Pu, one already at ambient conditions encounter distorted structures due to their substantial $5 f$-band occupation. In fact, as we proceed from $\mathrm{Pa}$ to $\mathrm{Pu}$, the $5 f$-band filling increases with about one electron per

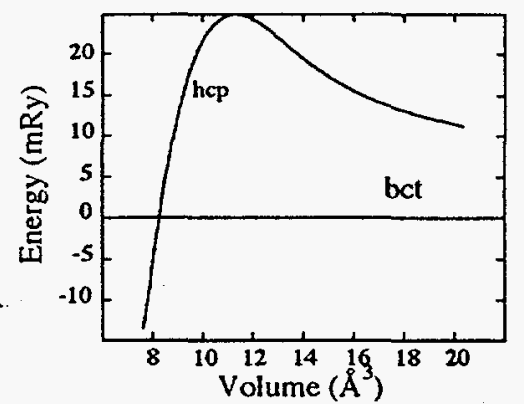

FIGURE 1 Total energy for Th in the hep structure relative to the energy of the bct structure, as function of volume.

element and the crystal structures display a pronounced increase of complexity. We have chosen here to study one of these metals more in detail, neptunium. Neptunium has one of the most complex crystal structures of the metals in the periodic table. In agreement with experiment, the ground-state crystal structure of $\mathrm{Np}$ is calculated (9) to be a simple orthorhombic structure with 8 atoms/unit cell $(\alpha-\mathrm{Np})$. Theoretically, a subsequent transition to the somewhat less complicated B-Np structure (orthorhombic with 4 atoms /cell), has been found (9). At even higher pressures neptunium transforms to the bec crystal structure, see Fig.2.

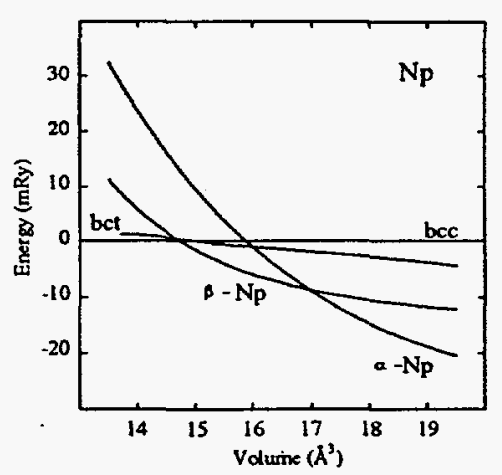

FIGURE 2 Total energy for $N p$ in the $\alpha-N p, \beta-N p$ and bct structure relative to the energy of the bcc structure, as function of volume. 


\section{DISCUSSION}

In the present report we focus on two actinide metals, namely thorium and neptunium. They serve as representatives for metals in the light actinide series, as regards their crystal structure behavior as a function of applied pressure. For Th, which have a relatively small number of $5 f$ electrons at ambient conditions, we have to apply a hydrostatic pressure (and increase the $5 f$ population) before we can make use of the general arguments for the crystal structure behavior we are to present. The other light actinides (Pa-Pu) have sufficiently large $5 f$ occupation for the $5 f$ band to become the most dominating band at ambient pressure.

As we have already mentioned, the crystal structures become open/complex (low symmetry) for the metals where the $f$ band dominates the bonding (compressed $\mathrm{Th}, \mathrm{Pa}, \mathrm{U}, \mathrm{Np}$, and $\mathrm{Pu}$ ). The reason for this behavior has been unclear until recently (1) when a link between the simple metals, the $d$-transition metals and the $f$-transition metals were discovered, namely the bandwidth of the dominating band. Hence, by manipulating the bandwidth in the calculations (by changing the lattice parameter) one could obtain both complicated structures (as in the actinides) or simple structures (as in the $d$-transition series) for all these different types of metals. The argument was (1) that for narrow bands close to the Fermi level $\left(E_{F}\right)$, the system could lower the total energy by lowering the symmetry of the crystal. Hence, degeneracies in the one-electron energies due to symmetry requirements of the crystal could be lifted so that weight is shifted from higher to lower energies in the electron density of states (DOS). This mechanism was referred to as a Peierles distortion (1) and was used to explain why the light actinides crystallize in low symmetry' structures.

This argument is most easily demonstrated for neptunium metal, even though it is generally valid for all the light actinides. In Fig.3. we illustrate the Peierles distortion by showing the calculated $5 f$ and $6 d$ DOS for Np for B-Np (left) and bcc (right) structures for a volume close to the equilibrium volume (lower) and a compressed volume (upper). First notice that the $6 d$ DOS is very low and featureless in the vicinity of the Fermi level, and that it is the $5 f$ and not the $6 d$ DOS that is dominating at higher energies. Notice also in the lower panel, that the $5 f$ DOS at the $\mathrm{E}_{\mathrm{F}}$ is much higher for bec than it is for B-Np. This is so since in the bce structure the symmetry of the crystal leads to degenerate levels close to $\mathrm{E}_{\mathrm{F}}$. However, for $\mathrm{B}-\mathrm{Np}$ the symmetry is much lower and many of these degeneracies disappear, and the $5 f$ DOS at $E_{F}$ is consequently lowered.

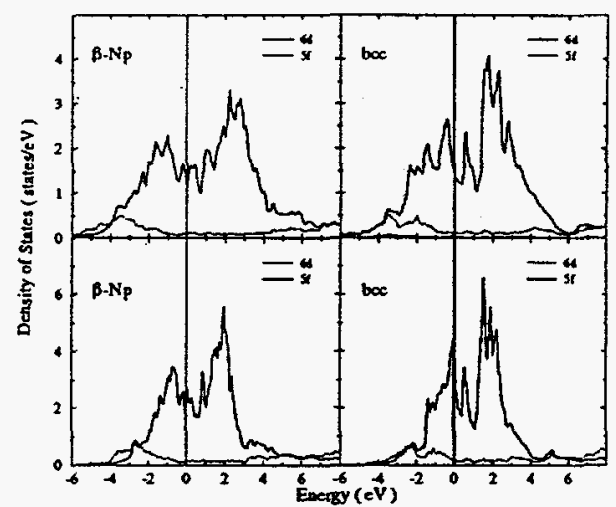

FIGURE 3 Calculated $5 f$ - and $6 d$-electron density of

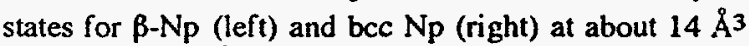
(upper) and $18 \AA^{3}$ (lower). Energies are in $\mathrm{eV}$ and the vertical lines indicate the Fermi level.

This symmetry breaking mechanism (Peierles distortion) lowers the one-electron contribution to the total energy and overcomes the increased electrostatic energy associated with the lowering of the symmetry of the structure. The balance between the one-electron energy and the electrostatic energy leads to that B-Np is favored over the bcc structure at the larger volume. When the metal is compressed the $f$ bands (together with all other bands) are broadened. The effect of this is obvious in Fig.3 (upper panel) where the difference in the DOS at the $E_{\mathrm{F}}$ has essentially vanished between the B-Np and bcc structure. Hence, for this volume, the Peierles distortion is no longer an efficient way to lower the one-electron energy and Np transforms to the bcc phase at this pressure due to the lower electrostatic energy of this structure. This transformation, $\mathrm{B}-\mathrm{Np}$ to bcc, is particularly interesting since one can define (approximately) a transformation path depending only on one internal parameter of the crystal structure. Hence it is possible to study this transformation in detail, as a function of pressure (9).

Another interesting question is of course why $\mathrm{Np}$ adopts the bcc structure at high compression. Nothing in the discussion so far has indicated that the bcc structure should be favored over, for 
instance, the fcc structure. Next, we will try to explain this with help of a canonical band picture for the $f$ bands. The following argument is much in the spirit of the explanation for the crystal structures of the $d$-transition metals, for which Skriver (10) used a canonical band description to analyze the observed hcp-bcc-hcp-fcc structural sequence through the (non-magnetic) $d$-transition metals. The canonical bands (11) are independent of volume and potential, and canonical in the sense that they are material independent which makes them very suitable to use for investigating band filling effects upon, for example, the crystal structure. Since they do not account for the electrostatic energy but only for the band (one-electron) energy they can only be used to compare crystal structures with a similar electrostatic energy, such as the fcc, hcp, and the bcc structures.

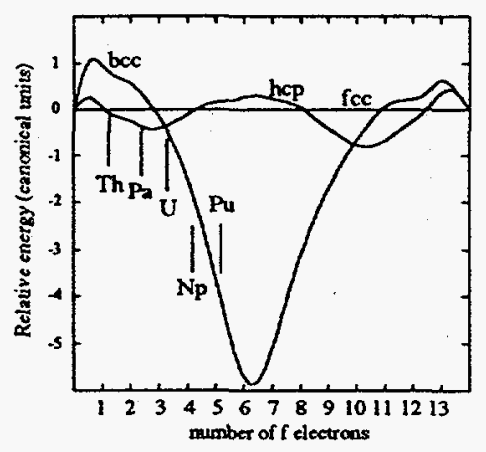

Figure 4 Canonical $f$-band energy differences (arbitrary units) as a function of $f$-band occupation. The fec canonical energy defines the zero energy level of the plot. Equilibrium $f$-band occupations for the light actinides (Th$\mathrm{Pu}$ ) are also shown.

In Fig.4 we show the canonical $f$-band energy as a function of $f$-band filling. For simplicity we plot energies relative to the fcc structure energy. Notice that for $\mathrm{Np}$ (which has about four $f$ electrons) the canonical energy is substantially lower for bcc than for both the hcp and fcc structures. We argue that this is the reason to that $\mathrm{Np}$ under compression eventually (when the $5 f$ band is sufficiently broad) crystallize in the bcc structure. Furthermore, this explains why all the calculated low temperature phases for $N p$ are more $(\alpha-N p)$ or less (R-Np) distorted bcc structures. The canonical band model can also be applied for other light actinides, such as Th for example. Figure 4 suggests that Th will transform to the hcp structure at ultra-high pressure, which our present electronic structure calculations indeed confirm. For $\mathrm{Pa}$ the model also predicts the hcp structure to be stable at high pressures whereas for $\mathrm{U}, \mathrm{Np}$, and $\mathrm{Pu}$ the bcc structure seems to be favored at large compression according to the canonical band theory.

\section{ACKNOWLEDGEMENTS}

We are grateful to Olle Eriksson, Börje Johansson and A.K. McMahan for valuable discussions. Work performed under the auspices of the U.S. Department of Energy by the Lawrence Livermore National Laboratory under contract number W7405-ENG-48.

\section{REFERENCES}

1. P. Söderlind, O. Eriksson, B. Johansson, J.M. Wills, and A.M. Boring, Nature, 374 , 524-525 (1995).

2. W. Kohn and L Sham, Phys. Rev. B 140, Al133A1138 (1965).

3. J.P. Perdew, J.A. Chevary, S.H. Vosko, K.A. Jackson M.R. Pederson, and D.J. Singh, Phys. Rev. B 46, 6671-6687 (1992).

4. P. Söderlind, O. Eriksson, B. Johansson and J.M. Wills, (Accepted for publication in Phys. Rev. B).

5. J.M. Wills (unpublished); J.M. Wills and B.R. Cooper, Phys. Rev. B 36, 3809-3823 (1987); D.L. Price and B.R. Cooper, Phys. Rev. B 39, 49454957 (1989); J. Trygg, Thesis, Uppsala University, (1995).

6. O.K Andersen, Phys. Rev. B 12, 3060-3083 (1975).

7. O. Eriksson, P. Söderlind, and J.M. Wills, Phys. Rev. B 45, 12588-12591 (1992).

8. Y.K Vohra and J. Akella, High Pressure Research, 10, 681-685 (1992).

9. P. Söderlind. B. Johansson, and O. Eriksson Phys. Rev. B 52, xxxx (1995).

10. H.L Skriver, Phys. Rev. B 31, 1909-1923 (1985).

11. H.L Skriver, The LMTO Method, (Springer, Berlin, 1984). 